\title{
Le joueur au travail ou le plaisir d'accumuler des richesses digitales
}

The Player at Work or the Pleasure of Accumulating Digital Wealth

\section{Stello Bonhomme}

\section{OpenEdition}

1 Journals

Édition électronique

URL : https://journals.openedition.org/sdj/1906

DOI : $10.4000 /$ sdj. 1906

ISSN : 2269-2657

Éditeur

Laboratoire EXPERICE - Centre de Recherche Interuniversitaire Expérience Ressources Culturelles Education

\section{Référence électronique}

Stello Bonhomme, "Le joueur au travail ou le plaisir d'accumuler des richesses digitales », Sciences du jeu [En ligne], 11 | 2019, mis en ligne le 18 avril 2019, consulté le 12 décembre 2022. URL : http:// journals.openedition.org/sdj/1906; DOI : https://doi.org/10.4000/sdj.1906

Ce document a été généré automatiquement le 12 décembre 2022.

\section{(c) (1) (9)}

Creative Commons - Attribution - Pas d'Utilisation Commerciale - Pas de Modification 4.0 International - CC BY-NC-ND 4.0

https://creativecommons.org/licenses/by-nc-nd/4.0/ 


\title{
Le joueur au travail ou le plaisir d'accumuler des richesses digitales
}

\author{
The Player at Work or the Pleasure of Accumulating Digital Wealth
}

\author{
Stello Bonhomme
}

1 Je voudrais commencer par quelques remarques simples, qui pourraient même passer pour simplistes, mais qui gardent un intérêt essentiel et qu'il faut absolument rappeler. Les jeux vidéo sont des fictions actives. En effet, si l'on devait isoler une détermination essentielle (pour ne pas dire fondamentale, au sens harmonique), ce serait sans doute cette possibilité, presque magique, d'altérer les images à distance, d'agir en un monde fictionnel. Nous pouvons même réduire cette détermination à l'illusion, car l'enfant qui s'escrime sur une borne d'arcade, incapable de comprendre ou refusant de voir la prescription INSERT COINS, s'illusionne de son influence sur les images qui défilent et dont il s'attribue le mérite. Il joue.

2 Plus sérieusement, l'émotion vidéoludique ressemble à celle de cet autre enfant que décrit Hegel (1998 [1835]) au début de son Esthétique, tout tendu vers la contemplation de son propre agir, occupé à faire des ricochets, à faire rebondir, ondoyer les images lointaines, sans avoir à se mouiller, ni besoin d'attraper des ampoules. La télé-action, ce n'est pas l'image qui est lointaine, c'est l'agir qui est médiatisé : communication. Sa médiation d'image, proprement illusoire, enlève toute nécessité de production utile, les mouvements ludiques ne sortiront pas de l'écran des images, proposant au joueur l'image d'un agir proche quant à la performance (influence directe), lointaine quant au résultat (autotélie).

En bref, le monde fictionnel n'est pas seulement décrit au moyen de textes ou d'images, mais d'un certain nombre d'actions possibles, prescrites par le logiciel. Il s'agit d'un basculement important de la fiction : du scopique - où l'image lointaine ne peut qu'être contemplée - au tactile, où l'image n'a de cesse d'être manipulée. Ainsi l'émotion du joueur n'est pas un simple mouvement en son âme, elle correspond à un mouvement musculaire, et devient le moteur d'une réaction. La question qui traverse l'ensemble de mes recherches en la matière est de savoir si l'image, la représentation au sens 
classique, peut survivre à la violence d'un tel basculement ou si le paradigme vidéoludique nous impose de retailler nos concepts.

Nous voudrions plus particulièrement nous intéresser ici aux affinités que peuvent entretenir les jeux vidéo avec le schéma de production du travail. Il nous faut insister sur le fait que notre étude, sa méthode comme ses produits, est proprement philosophique, et recouvre, plus spécifiquement le champ de l'esthétique. Nous ne prétendons pas à la mise au jour d'un modèle ludique général, ni de juger ou de faire la nomenclature de caractéristiques subtiles de jouabilité, mais simplement d'éclairer le concept d'image vidéoludique. Les jeux vidéo sont des objets fort épars dont l'unité conceptuelle n'a rien d'évident. Au-delà de l'interface informatique, ce qui rapproche Tetris (Mirrorsoft,1987) de Rogue (Epyx, 1980), de Faster Than Light - FTL (Subset Games, 2012) de Dance Dance Revolution (Konami, 1998), semble se réduire à un vague air de famille. Nous ne cherchons ni à recoller le vase, ni à réunir la famille de force pour dire ce que sont, ou doivent être, les jeux vidéo. En revanche, cela ne saurait nous conduire à nier la réalité d'un type très particulier d'images, de récits, de fictions, que produisent seulement les jeux vidéo, et à tenter d'en examiner les caractéristiques les plus saillantes. Le géomètre ne passe pas en revue l'infinité des triangles possibles pour savoir, d'un coup d'œil, que toujours la somme de leurs angles sera égale à deux droits. Notre objet n'étant pas le triangle, nous ne pouvons prétendre à la même universalité, comme forcés en outre de prendre le chemin inverse puisque ce sont les jeux vidéo eux-mêmes qui définissent l'image vidéoludique. Ce serait une entreprise tout aussi stérile qu'en géométrie, en revanche, de passer en revue tous les jeux vidéo pour y chercher, comme au hasard des rencontres, ce qui les distingue et fait consensus. Si notre discipline est la philosophie en ce que nous manipulons des concepts, notre approche doit être celle des sciences expérimentales et l'hypothèse que nous souhaitons mettre à l'épreuve et qui constituera notre point de départ, simple à l'excès, est celle du joueur au travail.

Prenant comme à contre-pied les études qui tendent à l'analyse de certaines techniques managériales à l'orée de processus de ludification, nous souhaitons examiner les jeux vidéo depuis le concept de travail et de production. Bien sûr que nous pouvons observer une influence des images vidéoludiques dans des domaines aussi divers que le management, l'éducation, l'architecture de sites et de réseaux sociaux, etc. Mais l'observation de cette tendance générale, aussi importante soit-elle, ne doit pas nous conduire à prendre le problème à l'envers. Si les images vidéoludiques influencent si bien nos manières de travailler, c'est peut-être précisément parce qu'elles consistent déjà elles-mêmes en des images de production. La notion de playbour (mot valise forgé à partir de play [jeu] et labour [travail]) pour la première fois théorisée par Julian Kücklich (2005) met l'accent sur une porosité essentielle entre l'action ludique et celle laborieuse et rejoint davantage nos préoccupations. Mais, en ce que son exemple privilégié réside dans les altérations du code d'un jeu par une communauté de joueurs pour améliorer voire créer des d'autres jeux (pratique de modifications d'un jeu ou modding), altérations qui viennent comme une aubaine, une manne de travail gratuit, pour l'industrie du jeu, pouvant s'épargner d'autant en développement et en marketing sans avoir à céder de droits aux auteurs/joueurs, la notion de playbour définit le travail par un profit réel, une valeur ajoutée.

6 Si nous aurons l'occasion de voir que nous pouvons faire la culture de données digitales, ces dernières pouvant même faire l'objet de transactions réelles, notre 
problème n'est pas économique et social mais esthétique. Ainsi, nous nous plaçons à un niveau bien plus modeste et nous nous contentons, pour notre part, de considérer le travail dans son procès, tel que Marx (1872) le définit au début du Livre I du Capital. Plus encore, nous ne mettons pas l'accent sur le caractère anthropologique de cette définition célèbre : comme Michel Serres (1980) nous retenons, en premier chef, que travailler c'est trier, lutter contre le désordre. Reste à savoir en quoi cela concerne l'activité du joueur.

7 Les jeux que nous examinerons en ce but ne seront pas choisis pour leur qualité vidéoludique: nos analyses porteront sur des jeux médiocres aux mécaniques grossières, comme sur de véritables réussites, sans nous intéresser à ce qui les distingue, ni même toujours relever la supériorité de l'un sur l'autre. L'enjeu n'est pas d'établir un critère de jugement pour reconnaitre les bons jeux vidéo, les mécaniques vertueuses de celles aliénantes, mais de mettre au jour ce dont il est question dans l'image vidéoludique, ce qu'elle nous donne à voir. ${ }^{1}$ En effet, la perspective du travail nous permet de court-circuiter le problème du statut narratif ou monstratif des images vidéoludiques pour lequel nous sommes insuffisamment armés si nous souhaitions le prendre de front. Au contraire, met-elle l'accent sur le caractère contraignant des signes chargés de prescrire des opérations, de donner des ordres au joueur, ainsi que sur toute une économie libidinale de l'effort et de la récompense.

Mais cette perspective n'a véritablement d'intérêt qu'à condition d'en sortir : une fois que nous aurons établi en quoi le jeu du joueur consiste en un simulacre de travail, il nous faudra savoir en tirer les conséquences du point de vue de la représentation. Car si le fait de produire est au centre de l'activité vidéoludique, le caractère tant fictionnel qu'autotélique de cette production nous impose de considérer le produit lui-même en tant que moyen et non en tant que terme. L'ensemble du processus comme circulaire, l'essentiel ne porte pas sur ce qui est fait (produit), mais sur l'image d'un faire (production), d'un geste permettant de reconstituer parmi les fragments de données, la continuité harmonieuse des images mobiles.

\section{Produire des richesses, obtenir des récompenses}

Mais n'allons pas trop vite et essayons de retrouver la belle simplicité de nos premières remarques. Le mouvement vidéoludique est nécessairement dirigé vers un but, pour qu'il y ait quelque ressort au jeu bien sûr, mais ne serait-ce que pour comprendre le mouvement qui va de $\mathrm{A}$ à $\mathrm{B}$, comme le signale Deleuze au début de L'Image-mouvement :

Si je considère des parties ou des lieux abstraitement $A$ et $B$, je ne comprends pas le mouvement qui va de l'un à l'autre. Mais [je le comprends] si je suis en A affamé et qu'en B il y a de la nourriture (Deleuze, 1983, p. 18).

Les jeux vidéo ont - en ce qu'ils demandent, prescrivent, des opérations à accomplir dans un certain ordre pour atteindre quelque produit - de grandes affinités avec le schéma de production du travail. ${ }^{2}$ Ainsi, le joueur rencontre toutes les émotions liées à l'exécution d'une tâche, le plaisir à être absorbé, tout tendu vers un but, la frustration de l'échec et la gratification du succès, souvent ponctuées à grand renfort de sons et d'images. Dans un grand nombre de jeux vidéo de rôle $(R P G)$, c'est la forme générale du roman d'apprentissage ou de la Bildung qui est adoptée. Le héros, faible au départ, doit augmenter sa puissance en réalisant des quêtes et en se battant contre des monstres ou d'autres joueurs, ce qui lui rapporte pièces d'or, objets magiques et autres points 
d'expérience, d'honneur, etc. Ce plaisir, lié à l'accumulation de richesses et de puissance, fournit une image rassurante de la progression et peut expliquer, pour partie, certains phénomènes de dépendances vis-à-vis de certains jeux vidéo qui n'en finissent jamais et où il est toujours possible d'obtenir un peu plus, d'augmenter davantage la puissance de son avatar.

11 Les travaux de Bluma Zeigarnik (1927) en psychologie montrent en effet qu'une tâche inachevée laisse une trace plus importante dans la mémoire, un sentiment d'incomplétude, qu'il s'agit de résoudre. Cet effet est également lié à la notion de persévération conative, c'est-à-dire la stimulation à achever une tâche maintenue par son inachèvement. Or, l'essentiel du jeu ne réside pas dans le voir, mais dans le faire. Ce que les jeux vidéo représentent, ce sont donc surtout des actions, des «faire machine avec ", l'image devenant outil, vecteur prescriptif fermé plutôt que contemplation ouverte. Ainsi, les images vidéoludiques apportent, comme sur un plateau, un ensemble de tâches inachevées de même que les moyens qui permettent, à coup sûr, de les achever. Ils sont de la même forme qu'un cercle en pointillés : il est bien difficile de résister à tracer les quelques traits qui manquent pour finaliser la tâche. De la considération d'une tâche inachevée naît une tension de l'esprit où l'action survient comme une détente, un plaisir.

Dès lors, il s'agit de distinguer deux choses. D'une part, le manque ou l'inachèvement en tant qu'impulsion et, d'autre part, le plaisir d'emplissage, ou de coloriage, où, au moyen d'un ensemble d'opérations prescrites par les propriétés d'usage (les tendances des images vidéoludiques), le joueur a l'impression d'accomplir quelque chose. Il perçoit des représentations d'actions et satisfait un besoin narcissique : celui d'utiliser les modalités de l'agir, de manière réglée, pour faire quelque chose, c'est-à-dire réduire la quantité d'information. Notons ainsi que les cinématiques de certains jeux ont, dans cette perspective, le rôle de flatter, une fois de plus, cette impression d'accomplissement en soulignant, d'une part, les grandes étapes d'avancement et de progression de la tâche et, d'autre part, une fois la tâche effectuée, autoriser un certain repos devant la tâche accomplie.

13 Ainsi, le joueur travaille, il réduit la quantité d'information, d'incertitude, pour ordonner le monde. Le programmeur, au sens très large d'auteur, propose toujours un puzzle d'images à remettre dans l'ordre. Il s'agit de la tension, l'enjeu qui fournit la raison de notre action, souvent rappelée au travers des journaux de quête, voire ramenée à une quantité (pourcentage de complétion du jeu, degré d'achèvement, etc.). Bien qu'il y ait d'innombrables subtilités, prenons un exemple excessivement simple et volontairement caricatural pour clarifier notre propos, celui que nous propose Edmund McMillen, célèbre designer de jeu indépendant, au travers du jeu A.V.G.M. (Mc Millen, 2010), jeu réalisé à l'occasion d'un concours de développement informatique en moins de deux jours. ${ }^{3}$

14 L'interface, simple à l'excès, propose un seul bouton. Le cadre, géométrique, se divise en blocs rectangulaires, une pièce comme un enfant pourrait la dessiner, sol, mur et fenêtre. À droite, un interrupteur. Un bloc, sous forme de phylactère, zoome sur ce dernier et prescrit les tâches à accomplir : pour faire apparaître des objets dans la pièce, il nous faut cliquer. Un compteur est d'ores et déjà là pour recueillir le score. Lorsqu'on clique effectivement, la lumière s'éteint, plongeant la pièce dans le noir, on clique à nouveau, elle se rallume, laissant parfois apparaître derrière une poussière d'étoiles et un échantillon sonore, un élément de mobilier, ou tout du moins un objet, 
table ou chaise, que l'on peut déplacer à l'envi dans la pièce. Le nombre de clics nécessaires à l'apparition de nouveaux objets croît exponentiellement. Il y a 41 objets à débloquer. Après que tous les objets sont apparus, ils se colorisent. Pour finir le jeu, il faut cliquer dix mille fois sur l'interrupteur, rien de plus.

L'auteur interrogé sur son jeu dénonce la supercherie: «A.V.G.M. était une plaisanterie", l'acronyme signifie Abusive Video Game Manipulation [Manipulation vidéoludique abusive]. L'interrupteur utilisé pour débloquer les objets est une allégorie des actions extrêmement répétitives et simples qui absorbent le joueur, désireux de finir le jeu ou de passer au niveau supérieur. A.V.G.M, plus encore, cherche à dénoncer les abus de cette technique de design de jeu qui joue sur la compulsion d'achèvement et d'accumulation, technique qui se fait tactique commerciale, permettant de rendre le joueur accroc à moindre frais, en jouant grossièrement sur l'impression d'avoir accompli quelque chose, par la pression compulsive de boutons qui actionnent le déblocage de trophées ou de médailles.

Si le jeu est un travail, la récompense, même digitale, joue un rôle psychologique primordial. Elle est le produit de l'agir du joueur et active le système de récompense et de renforcement. Les images nous dressent à agir, presque comme dans une expérience pavlovienne. Il y a quelque chose d'abusif car les joueurs se trouvent véritablement bloqués dans un comportement stéréotypé. Bien qu'il s'agisse ici d'un cas-limite, d'une caricature, force est de constater que de nombreux jeux vidéo et plus particulièrement les jeux de rôle et les jeux de rôle massivement multijoueur fonctionnent, au moins en partie, sur ce modèle. De même les jeux gratuits (avec un modèle économique reposant sur du contenu additionnel payant - souvent nommés en anglais free-to-play ou plus lucidement pay-to-win - proposent souvent une jouabilité similaire. Si les récompenses sont en nombre suffisant, graphiquement valables et s'inscrivent dans la continuité d'un univers, on peut faire un jeu vidéo rentable ${ }^{4}$ où il suffit d'appuyer pour collecter des données, cliquer sur des portions de l'image pour augmenter son score, décorer le bateau des pirates, élever des œufs de dragons, etc.

C'est une technique [de jouabilité] utilisée dans tous les MMORPG, qui consiste en une manière affreuse et abusive d'accrocher [hook] des gens à votre jeu et une chose incroyablement minable [lame] à faire. Les avantages de cette technique sont évidents, vous pouvez facilement accrocher du monde, faire payer des gens pour jouer, et aussi longtemps que vous les ligotez avec toujours de nouvelles déclinaisons [expansions] de la même situation, vous pouvez tout simplement les saigner à blanc sans jamais rien ne leur donner en retour (notre traduction)..$^{5}$

En effet, si l'on télécharge en ligne le jeu Dofus (Ankama Games, 2004) gratuit - ou bien plutôt qui repose sur un modèle économique d'achats intégrés - on sera d'emblée plongé dans des processus d'accumulation. Il nous faudra, par exemple, cliquer sur un épi de blé pour récolter la ressource et gagner des points d'expérience (XP) dans un domaine, cette même opération répétée, déclinée à l'envi. Plus profondément, tous les éléments du jeu, sous couvert d'un habillage fantaisie médiévale (heroic fantasy), consistent en une économie de valeurs numériques. L'accumulation de ces valeurs et notamment le Kama, unité monétaire générale du jeu, ainsi que l'XP, demande un investissement chronophage. Des vidéos, des forums sur internet sont consacrés au développement de techniques pour accumuler le plus de Kama, nous en reparlerons car il s'agit déjà de stratégie et de rationalisation du travail.

Restons-en pour le moment à la structure compulsive du clic efficace. En l'espace d'un mois et vingt jours, Edmund Mc Millen a recensé, sur huit mille joueurs, trois cent 
trente-sept qui ont battu le jeu, c'est-à-dire qui ont cliqué dix mille fois sur l'interrupteur. Même en admettant qu'il y en ait quelques-uns qui aient triché en utilisant un algorithme qui va cliquer automatiquement (de type autoclicker) - ce qui leur a demandé un effort de toute manière -, quatre pour cent reste une proportion significative, au vu de la médiocrité ludique assumée et surtout de l'extrême redondance de l'action proposée. Mais on ne peut pas perdre, on progresse, et il nous est difficile de quitter une tâche qui progresse, aussi médiocre soit-elle, nous nous y sommes attachés. Tout est fait pour nous accrocher, une multitude de sons et d'images déployées, toute une fanfare qui applaudit nos clics et renouvelle constamment la promesse d'une récompense. Au début, on progresse vite comme dans les jeux de rôles où les niveaux d'expérience sont organisés sur une échelle exponentielle. Quand cela devient trop long, il est déjà trop tard, on a trop investi pour abandonner. De plus, on accorde d'autant plus de valeur à la récompense attendue, le nouveau sort, la nouvelle pièce d'armure, que cette dernière demande d'autant plus d'efforts répétitifs. ${ }^{6}$

Concentrons-nous sur le caractère psychologique de ce désir d'accumulation et de progression, désir qui s'accomplit dans un geste compulsif, sensiblement auto-érotique. Dans Final Fantasy VII (Squaresoft, 1997), comme dans la plupart des jeux de ce genre, il y a trois phases de jeu principales : les villes, le déplacement sur la carte du monde, les donjons. Dans les deux dernières, une tâche extrêmement répétitive est la somme abrutissante de combats qui interrompent notre route, combats générés aléatoirement, et qui surgissent brusquement. On échange ses ressources à la ville, on les collecte en chemin. Le combat lui-même obéit à des règles au tour par tour, selon le même principe du jeu de rôle sur papier, c'est-à-dire un combat où l'on va générer des points de dégât en lançant des sorts, des dés. À la fin de ces combats, on fait le décompte des valeurs, combien d'XP, combien de Gil, quelle potion, quel objet a-t-on pu récupérer. Les points d'XP permettent de faire progresser les personnages : au bout d'une certaine quantité de points, ils augmentent de niveau (to level up) et lancent des sorts plus puissants. Tout peut être ramené à un ensemble de valeurs quantifiables à faire prospérer, valeurs qui forment l'identité du personnage, ce qu'il est, ce qu'il possède.

Dans les jeux de rôle en ligne, massivement multi-joueurs, tel que World of Warcraft (Blizzard Entertainment,2004) ou encore Dofus, le fait que le joueur fasse presque toujours la même chose est compensé par le fait qu'il ne cesse jamais de progresser. Il est étonnant de remarquer à quel point cela peut fonctionner, l'on est très vite pris dans cet engrenage de l'action automatique efficace. Le désir de faire monter son personnage est important, même si cela implique un lourd investissement de temps, où une tâche rébarbative et aliénante, digne de l'organisation scientifique du travail, sera répétée à l'excès. Il est profondément ironique de voir que le verbe employé pour désigner cette action du joueur purement dirigée vers l'accumulation de denrées digitales (or, expérience, etc.) soit issue du travail : le farming. En effet, le joueur ne joue presque plus, il rationalise les moyens de production, délimite une zone de farming où il trouvera ce qu'il souhaite en abondance, or, butin à piller, points d'expérience ou encore matière première à transformer pour produire de nouveaux objets (ce qu'on appelle le crafting). On remarque, à ce propos, qu'il existe même une profession de farmers professionnels en Chine, chargés de jouer plusieurs heures par jour, pour récolter des récompenses digitales (or, armes, etc.) et de les revendre ensuite illégalement sur internet. Ce ne sont plus des joueurs, ce sont des ouvriers, la différence essentielle ne se situe que sur un plan psychologique mais elle est de taille. 
21 Ainsi, si l'action devient vraiment trop répétitive, l'on peut, acheter des pièces d'or à ces farmers. Une fois la transaction établie en argent réel sur internet, on prend livraison de la commande par l'intermédiaire de la boîte aux lettres du jeu.

Le prix réel de chaque chose, ce que chaque chose vaut réellement pour celui qui l'a acquise et qui cherche à en disposer ou à l'échanger pour quelque autre objet, c'est la peine et l'embarras que la possession de cette chose peut lui épargner et qu'elle lui permet d'imposer à d'autres personnes. (Smith, (1999 [1776]) p. 38)

Ce que le joueur achète, c'est du temps de travail comme chez Adam Smith (1999 [1776]) dans Richesse des nations, il s'épargne la peine de jouer, l'illusion de l'agir est si forte dans son effectuation automatique qu'elle a donné une valeur réelle à des simulacres digitaux, une suite de signes formant code binaire. Il peut également créer un algorithme, un robot chargé d'œuvrer pour lui. Alors même que le seul intérêt était de jouer, le fait que la tâche soit répétitive et contraignante, augmente la valeur de la denrée digitale «Pièce d'or » ou de tel autre item plus ou moins difficile de piller (plus connu en anglais sous «loot ${ }^{7} »$ ), échangeable sur un marché économique intra diégétique (à condition que le jeu le permette). Tous ces exemples nous montrent combien le joueur accorde de l'importance aux objets qu'il s'approprie par les opérations ludiques prescrites par le jeu, même quand ces dernières sont simples et répétitives. On pourrait sans peine nous objecter que tous les jeux vidéo, tant s'en faut, ne reposent pas sur un mécanisme d'appropriation, et qu'un grand nombre ne permet même aucune forme d'accumulation. Il s'agirait également de distinguer les récompenses extra diégétiques (médailles, scores, "achievments ", etc.) et les denrées digitales intra-diégétiques (pièces d'or, objets magiques, etc.).

\section{L'ouvrier des images}

23 Mais là n'est pas l'essentiel de notre propos. Contentons-nous de remarquer qu'il est possible de réduire à l'extrême interface et jouabilité, ou, plus simplement, l'ensemble des opérations prescrites (cliquer et collecter), à condition que l'image d'une production ou d'une progression satisfasse suffisamment les efforts du joueur, qu'il soit payé en retour en ayant produit quelque chose. L'accumulation, la transformation et la gestion ne sont que des types de production parmi d'autres. Aucun jeu ne consiste à ne pas agir, à ne pas exercer d'influence sur ou à ne pas faire. Même une narration interactive, structurée en arbre de choix, voire sous forme de "simulateur de marche " comme dans Gone Home (The Fullbright Company, 2013) ou dans un monde dit ouvert, n'échappe pas à la progression nécessaire du jeu.

Il existe un jeu indépendant fort populaire, The Stanley Parable (Galactic Café, 2013), qui traite fort justement de ce thème. Il s'agit de l'histoire de Stanley, employé de bureau, heureux dans son travail qui consiste, pour l'essentiel, à appuyer sur les boutons de son clavier en fonction des ordres reçus sur son écran. Mais, un beau jour, il ne reçoit plus d'ordres et se rend subitement compte que tous ses collègues ont disparu. Le joueur prend alors le contrôle de Stanley qui, après être resté longtemps interdit, se décide enfin à sortir de son bureau pour s'aventurer dans l'open space et les couloirs désertés. Dans un environnement 3D en vue à la première personne, nous sommes toujours accompagnés d'une voix off, un narrateur chargé de guider Stanley vers l'histoire, cette dernière se modifiant en fonction des choix du joueur. Le jeu présente une vingtaine de fins alternatives dont aucune n'est pleinement satisfaisante. La marge de manœuvre est 
particulièrement étroite : aucune porte fermée ne s'ouvre, on ne peut interagir avec presque aucun élément. Les alternatives sont simples et n'impliquent pas de critères moraux, ils se contentent de questionner la narration en arbre de choix. Le joueur rencontre par exemple deux portes, une à droite, une à gauche tandis que le narrateur déclare « Stanley prit la porte de gauche».

Dans une combinaison de choix où le joueur résiste constamment à faire ce que dit le narrateur, ce dernier redémarre le jeu et finit par mettre en place un nouvel élément de design de jeu : "The Stanley Parable Adventure Line», ou une simple ligne jaune pour aider le joueur à ne plus se perdre. La tonalité épique que prend soudain la musique alors que nous traversons les couloirs en suivant cette ligne souligne le caractère parodique qui, plus profondément, consiste en une allégorie des jeux vidéo : le chemin est tracé à l'avance comme en pointillés et tend vers son achèvement. La mise en abyme de l'écran de titre est on ne peut plus claire, Stanley, comme le joueur, est prisonnier du jeu. Quoiqu'il arrive, malgré toute la profusion de branches, la confusion même de ces branches, le jeu progresse nécessairement vers son terme, vers l'un de ces termes, le joueur ne peut pas faire autre chose que de suivre des flèches.

L'expérience réalisée sur le réseau social Twitch avec le jeu Pokémon (Nintendo, 1997) le montre également avec force ${ }^{8}$. Qu'il s'agisse de répondre avec satisfaction à la requête de l'écran en appuyant sur les boutons qui correspondent sur le clavier (le travail de Stanley) ou de suivre un chemin, il s'agit toujours de se soumettre à des prescriptions pour progresser, faire fonctionner ou dérouler le jeu. La narration est produite au sens où elle dépend d'une action ou d'une suite d'actions, son progrès dépend de la soumission du joueur aux prescriptions, s'il accepte de se faire ouvrier des images. Ce en quoi The Stanley Parable déroute et se trouve dans la continuité d'A.V.G.M. (une fin alternative nécessite d'appuyer sur un bouton sous les railleries du narrateur pendant quatre heures pour être débloquée), c'est qu'il ridiculise cette appétence pour la complétion d'une tâche déjà virtuellement accomplie. La ligne finit par se perdre dans une histoire sans queue ni tête et ne nous dévoile que l'absurde du chemin parcouru.

Toujours est-il que la structure binaire du code informatique est particulièrement propice à la mise en scène de la réduction d'une certaine quantité d'informations virtuellement présente qui ne demande qu'à être actualisée. Voilà l'origine du caractère compulsif. Mais que la compulsion soit comme inscrite dans la structure même du jeu ne veut pas dire que tous les jeux sollicitent des mécaniques psychologiques peu loyales pour accrocher l'attention du joueur au point de lui faire réaliser des tâches sans intérêt. Il est indéniable que certains jeux exploitent davantage ce caractère compulsif que d'autres par le moyen d'une déclinaison de tâches répétitives et de beaucoup de récompenses, ou par l'impossibilité de remplir la tâche pourtant à portée sans recourir à un "pay-to-win». De même, lorsqu'il ne s'agit plus d'un monde fictionnel limité dans le temps mais d'un « univers persistant » sans fin, où les tâches n'ont de cesse de se renouveler voire de ne jamais cesser de promettre un achèvement, tâches sinon simples, au moins à la portée de tous, le joueur peut se faire juif errant occupé à performer le néant pour y accumuler nombre de distinctions digitales dont il est fier et qu'il regarde comme le fruit mérité de son dur labeur.

Sans jeter l'opprobre sur telle ou telle autre technique de jouabilité, puisque ce serait comme négliger la notion d'expérience vidéoludique qui donne au joueur une responsabilité (on peut très bien jouer à World of Warcraft sans faire de farming), nous pouvons proposer, très provisoirement, de définir le caractère aliénant d'un jeu d'une 
manière semblable au travail. Pour aller vite, ce qui a été dérobé à l'ouvrier sur la chaîne de montage, c'est la conscience de finalité et l'ensemble de la séquence ordonnée. Il ne construit plus la table, il visse le boulon et c'est la répétition de ce geste qui va jusqu'à le dessaisir de ses propres mains. Le problème est bien différent et moins tragique pour ce qui concerne les jeux vidéo, mais il n'en reste pas moins similaire.

Si les images vidéoludiques prescrivent des moyens pour réaliser des tâches (en d'autres termes actualiser des images virtuelles), toute la question est de savoir le "jeu ", au sens technique de distance de la déformation des pièces d'un serrage par rapport à leur état libre, entre ces moyens et leur effectuation. Lorsque ce jeu, cette distance, se rapproche de zéro comme dans A.V.G.M., le joueur visse des boulons, répète, collecte, déroule, retenu qu'il est, artificiellement par quelque cosmétique qui donne du change aux efforts et par des récompenses qui fournissent l'essentiel de l'image de la progression, de l'efficacité du joueur. En revanche, lorsque le jeu se fait plus lâche, que le joueur doit découvrir par lui-même comment répondre aux prescriptions de manière satisfaisante, donner de l'ordre aux images ou apprendre à correctement manier son personnage, l'effectuation de la tâche elle-même peut constituer la part la plus importante de la récompense.

\section{La production désirante}

Pour qu'il y ait satisfaction de soi, il faut en effet rencontrer une certaine résistance, que l'agencement des images ne soit pas simple, qu'il requière de l'adresse, une certaine agilité, une intelligence déductive ou stratégique, voire même, comme nous l'avons vu, un certain degré d'acharnement. Les distinctions accordées, les scores, sont là aussi pour distinguer les joueurs par leurs performances et, par là même les individualiser, leur faire comme une place à l'intérieur du jeu (la marque du tableau de score). En ce sens, la partition vidéoludique n'est jamais pleinement linéaire, il existe presque toujours plusieurs degrés d'accomplissement, plusieurs distinctions et récompenses.

Pour prendre un exemple parmi mille, nous pouvons évoquer les différents degrés d'accomplissement d'un jeu de plateforme très linéaire comme Crash Bandicoot (Naughty Dog, 1996) avec les distinctions accordées (comme les Gems) en fin de niveau lorsque toutes les caisses ont été brisées. L'acquisition de toutes ces distinctions permet de découvrir une cinématique, une fin alternative. On dit de ces tâches "facultatives " qu'elles rallongent «la durée de vie » du jeu. Mais au-delà, la multiplication, ainsi que la complexité plus ou moins importante des tâches, montrent bien que l'intérêt du jeu réside dans l'accomplissement de la tâche, dans le parcours et non le chemin parcouru. La gestion de la difficulté constitue, en ce sens, un des aspects les plus essentiels dans le développement d'un jeu car ce qui rend, en tout premier lieu, le jeu appétant n'est pas la liberté de faire ce que l'on souhaite en un monde fictionnel, mais bien de pouvoir se soumettre à des règles pour accomplir avec plus ou moins de réussite une tâche : un travail.

Dans le jeu indépendant de Lucas Pope, Papers Please (3909 LLC, 2013), le joueur incarne un douanier chargé de vérifier les papiers des personnes qui se présentent au postefrontière de Grestin pour entrer en Aristoska, pays fictionnel appartenant au bloc de l'Est en pleine guerre froide (1983). En apparence, rien de plus ennuyeux. D'autant que si, dans le cours de la narration, quelques événements sporadiques viennent briser la 
routine (rencontres avec un mouvement résistant ou un migrant haut en couleur qui conduisent à des choix moraux, attentats qui donnent l'occasion d'un mini-jeu de tir...), l'essentiel du jeu consiste à effectuer cet ouvrage ingrat de fonctionnaire, et de tamponner avec justesse les passeports pour ne pas recevoir de réprimande du ministère des admissions. Ainsi, le joueur doit-il savoir appliquer les règlements et consignes en vigueur, connaître les préfectures capables d'émettre des passeports dans les pays voisins (Yurko en Koléchie par exemple), savoir à quoi ressemblent les différents sceaux diplomatiques, etc., toutes ces informations étant naturellement consultables à l'intérieur du jeu par l'intermédiaire d'un livre sur le bureau du douanier. Au début, on ne sait pas très bien comment chercher parmi toutes ces informations, d'autant plus que la place dont on dispose sur le bureau est fort limitée. Les réprimandes, puis les amendes, peuvent très vite s'accumuler. Mais tout l'intérêt réside justement dans cette importante difficulté. Il faut apprendre à s'organiser, trouver des méthodes de vérification pour ne pas passer à côté d'une anomalie. On ne tarde pas à se rendre compte que le jeu exige la rigueur et l'observation la plus attentive et à prendre goût à cette exigence formidablement bien mise en scène.

Nous avons évoqué plus haut les stratégies que l'on peut trouver sur internet pour accumuler beaucoup de « kamas », unité monétaire du jeu Dofus. On peut railler leurs efforts, mais il n'en reste pas moins que la plupart de ces tutoriaux ne manqueraient pas d'étonner le néophyte, et le propos hors de tout contexte pourrait être pris pour d'étranges conseils d'investissements financiers.

On peut trouver dans la retranscription d'une de ces "combines " intitulée "Se faire entre 20 et $40 \mathrm{M}$ de kamas par semaine en étant mono-compte $»^{9}$, tout le vocabulaire du travail, de la contrainte qui se trouve résolue par une certaine médiation, un savoirfaire où l'on doit respecter les étapes («Mon élevage, c'est de l'élevage puis transformation des dragodindes en parchemins de caractéristiques et enfin, revente des parchos»). C'est donc une séquence ordonnée et rationnelle, à la recherche de l'optimisation des valeurs récoltées. Ce trait est particulièrement visible dans les jeux de gestion où il s'agit d'organiser les flux de valeurs de manière optimale. Le joueur n'est pas simple ouvrier spécialisé, il se fait architecte, ingénieur, urbaniste et dans un jeu tel que Rimworld (Ludeon Studios, 2018), il faut, au bas mot, une vingtaine d'heures d'apprentissage pour maîtriser correctement les stratégies de base.

Le joueur n'exerce donc pas un fiat libre. En contrôlant l'image-sujet, il se branche sur les tendances qui conduisent les images du jeu vers une fin et se trouve, ainsi, presque autant contrôlé par les images, que lui-même les contrôle. Le joueur forme un couple avec les images dans le but de produire quelque chose. Ainsi, sommes-nous en plein dans le modèle deleuzien du désir où il s'agit de processus de production, un accouplement de deux machines, un « faire machine avec » :

Les machines désirantes sont des machines binaires, à règle binaire ou régime associatif ; toujours une machine couplée avec une autre. La synthèse productive, la production de production, a une forme connective : « et ", " et puis "... C'est qu'il y a toujours une machine productrice d'un flux, et une autre qui lui est connectée, opérant une coupure, un prélèvement de flux (le sein - la bouche). (Deleuze et Guattari, 1972, p. 11)

Une machine productrice d'un flux continu d'images en mouvement est couplée sur une machine chargée de couper le flux, d'y mettre un ordre. En ce sens, le désir ne se trouve plus assujetti à un besoin ou un manque, il se réalise dans le juste équilibre des 
deux machines. Comme nous le suggère une partie de la théorie lacanienne du désir, il est production réelle, dépassant toute idée de besoin ou de fantasme. avons vu qu'on ne peut pas réduire l'expérience vidéoludique à la compulsion d'achèvement et d'accumulation décrite par A.V.G.M. En revanche, il y a déjà dans A.V.G.M., quelque chose d'une rythmique, d'une gestuelle que le joueur imprime dans les images. Jour, nuit, le cliquetis, les jeux d'apparitions et de disparitions, les images 
qui se déroulent au fur et à mesure de l'action du joueur. Il y a même dans la répétition caricaturale de cette action quelque chose de plus profond: c'est par la répétition de l'action, ou d'un certain type d'action, que le joueur rythme le flux continu. Et c'est de la répétition que naît le rythme.

Les jeux vidéo sont, au même titre que le cinéma, des systèmes qui reproduisent le mouvement. Nous pouvons remarquer la parenté entre la machine de Turing et le projecteur cinématographique. Dans de nombreux jeux vidéo, nous avons l'impression qu'il s'agit même d'un système qui fonctionne comme le cinéma, à la différence près que, parmi des images qui bougent, il y a des images que je bouge. Nous revenons à notre première remarque, en la caricaturant encore un peu: «le jeu vidéo, c'est du cinéma actif ", du cinéma sur lequel j'ai une emprise directe.

Ce raisonnement, bien que fautif, n'est pas pour autant dénué d'intérêt. En effet, la mobilité des images vidéoludiques ressemble, du moins de l'extérieur, en de nombreux points, à la mobilité cinématographique, et nous pourrions presque dire que Deleuze semble le suggérer lorsqu'il déclare, dans L'image-mouvement, que «si le cadre [cinématographique] a un analogue, c'est du côté d'un système informatique plutôt que linguistique » (Deleuze, 1983, p. 23). Il y a dans les jeux vidéo, comme au cinéma, un cadre, "un système clos, relativement clos, qui comprend tout ce qui est présent dans l'image " (p.23), qui comprend donc un certain nombre de parties et de sous-parties (images) en mouvement. Ce qui, pour Deleuze, fait l'essence du cinématographique semble même être, au premier abord, entièrement respecté. En revanche, l'originalité cinématographique est d'être " un système qui reproduit le mouvement en fonction $d u$ moment quelconque » (p.13), c'est-à-dire en fonction d'instants équidistants choisis de façon à donner "l'impression de continuité ", les fameuses vingt-quatre images par seconde pour aller vite. Et bien loin de reconstituer artificiellement le mouvement par une simple succession de coupes immobiles comme le pensait Bergson méfiant vis-à-vis du cinéma, l'équidistance des instantanés transforme la succession de coupes immobiles en coupes mobiles, en images-mouvements, retranscrit le mouvement en tant que ce qu'il est, c'est-à-dire en tant qu'Ouvert, toujours en train de se faire.

À l'inverse, tout système qui conçoit le mouvement comme le passage réglé d'une forme à une autre: c'est-à-dire un ordre de poses [de thèses] ou des instants privilégiés, comme dans une danse [...] tout autre système qui reproduirait le mouvement par un ordre de poses projetées de manière à passer les unes dans les autres ou à se transformer, est étranger au cinéma. (Deleuze, 1983, p. 13).

Le cinéma c'est l'instant quelconque, l'équidistance des instantanés, la conception scientifique moderne, plutôt que la succession des instants privilégiés (la conception antique). La conception du mouvement vidéoludique semble au premier abord être la même que la conception cinématographique. Le jeu vidéo peut, après tout, s'apparenter au genre de l'animation par ordinateur qui, tout comme le dessin animé, appartient pleinement au cinéma en tant que l'animation » n'y constitue plus une pose ou une figure achevée, mais la description d'une figure toujours en train de se faire et de se défaire, par le mouvement de lignes et de points pris à des instants quelconques de leur trajet » (p.14). Mais le cadre cinématographique est un cadre, par essence, passif - un cadre sur lequel je projette une image - tandis que le cadre vidéoludique est un cadre informatique, dynamique, sur lequel je peux imprimer un mouvement. La comparaison la plus superficielle d'une session de jeu vidéo avec une séquence cinématographique ne peut pas ignorer le caractère davantage heurté, fragmentaire du jeu vidéo, et ce plus encore lorsque le joueur est inexpérimenté. Le modèle des jeux vidéo, ce n'est pas le 
cinéma et le couple projecteur/toile blanche mais bien l'oscilloscope, comme nous l'apprend l'anecdote de la création du prototype Tennis for Two, ou, les premiers modèles de caméra-projecteur. Lev Manovich (2006) insiste sur la double hérédité de l'ordinateur, tout à la fois tributaire du daguerréotype et de la machine analytique de Babbage. Cette double hérédité de l'ordinateur conditionne la dualité essentielle des images vidéoludiques, tout à la fois data et picture, image et donnée.

Ainsi, le fait qu'il n'existe pas seulement des images qui bougent et que je reçois passivement, mais également des images que je bouge volontairement, celles-ci enchevêtrées avec les premières, introduit une différence réelle: ce n'est plus le mouvement, c'est le geste, une rythmique qui est en question. En effet, les images actives (Mario) étant enchevêtrées, indémêlables des images passives (le reste du monde fictionnel, la structure cinématographique de l'équidistance des instantanés), ce n'est plus seulement le mouvement continu des images qui importe mais la mise en relation de deux types d'images: actives et passives. Il y a, dans le jeu vidéo, une rencontre entre deux types d'images mobiles, entre deux types de mouvements, au sein d'un même système, rencontre qui conditionne le déroulement de la fiction.

La rencontre entre l'image active et l'image passive doit se faire au moment juste, sur la bonne frame, c'est pourquoi bien plus que de mouvement, il s'agit de rythme. Au milieu de ce système continu analogue au cinéma, avec une équidistance des images quelconques, se fonde une structure de la rencontre de deux pôles, de la pulsation, du rythme, de la succession de poses discontinues et d'instants privilégiés. Il s'agit, pour le joueur, de dénicher, au milieu du mouvement continu, l'instant privilégié : voilà le travail du joueur et son plaisir. Il doit organiser les flux dans une rythmique, et que ce soit le plus harmonieux possible.

Pour bien comprendre cette idée de rythme, commençons par prendre pour exemple deux types de jeux vidéo où ceci apparaît de façon particulièrement nette : le jeu de plates-formes et le rythm game, avant de l'éprouver là où la notion semble davantage contre-intuitive.

Dans un jeu de plates-formes, l'accent principal est mis sur l'habileté qu'a le joueur à contrôler son personnage. Le jeu le plus emblématique de ce genre est, sans doute, la saga des Mario avec notamment Super Mario Bros. sur la console Nintendo Entertainment System (NES). Il s'agit essentiellement d'aller d'un point A (départ du niveau) à un point B souvent matérialisé, que ce soit par un drapeau (Mario), un panneau (Rayman) ou encore un tourbillon (Crash Bandicoot), en restant en vie. Rester en vie signifie le plus souvent ne pas tomber, ne pas être touché par un monstre ou un piège. Un jeu de plates-formes est fait de pièges à déjouer en fonction du temps et du rythme. Les monstres avancent selon un trajet déterminé à vitesse constante, la lave décrit des arcs de cercle constants ${ }^{10}$. En bref, il est possible de comprendre la structure des mouvements des pièges, car il s'agit de patterns répétitifs. Le joueur devra faire avancer son personnage en rythme, le faire sauter sur la plate-forme à tel moment et pas à un autre, à un instant privilégié.

La difficulté du jeu s'accroît au fur et à mesure des niveaux, l'intervalle durant lequel le personnage doit sauter se fait de plus en plus court et le joueur aura de moins en moins de temps pour observer et deviner à quel instant et avec quelle intensité il doit sauter. La fenêtre rétrécit. Ce qui est imposé par le jeu, c'est ce qui est imposé par les images passives à structure continue sur lesquelles je n'ai pas d'emprise directe et que je dois 
rencontrer successivement dans le bon ordre des poses, dans la pulsation rythmique correcte des instants privilégiés.

Encore davantage significatifs, les jeux de rythme (rythm games) ou les jeux de danse sont des jeux où il ne s'agit que de rythme. Le joueur voit à l'écran des images représentant les boutons de son contrôleur converger vers un point à une certaine vitesse sur une ligne de temps et il doit appuyer sur les boutons de son contrôleur à l'instant où, sur l'écran, les images rencontrent le point. Ni avant, ni après, à l'instant même.

51 Mais peut-on induire de ces jeux, dont la caractéristique première est justement d'exploiter ces ressorts d'urgence et d'adresse, une idée plus générale de gestuelle et de rythmique qui puisse s'appliquer à l'ensemble des images vidéoludiques? Ce qui nous importe ce n'est pas que la cadence imposée se réalise de manière temporelle, ni linéaire, mais que ce soit par la mise en relation des images fragmentaires, des données éparses, que le joueur conditionne les transformations, le déroulement, la progression de la narration. Les jeux de rôle, qui nous ont occupés plus haut, sont davantage tributaires d'une rythmique spatiale qui passe par l'exploration de territoires, l'accumulation de denrées digitales et l'organisation de ces dernières. De même, pour les jeux narratifs à animations pré-calculées comme Myst où, à l'image d'un casse-tête chinois, le joueur doit « remettre de l'ordre » dans le puzzle de données et d'images. Il doit, en quelque sorte, retrouver l'ordre des poses privilégiées d'un espace continu.

Dans le jeu de Lucas Pope, Return of the Obra Dinn (3909 LLC, 2018), le caractère fragmentaire de l'image et de la narration vidéoludique est exploité avec une grande justesse. Le joueur doit mener l'enquête sur l'identité et le destin de tous les passagers et membres de l'équipage d'un bateau d'où personne n'est revenu. Armé d'une montre magique, à chaque fois que nous rencontrons un cadavre sur le bateau, nous avons accès à l'instant figé de sa mort, et c'est à partir de ces informations partielles que nous devons retrouver le fil de la narration. Il s'agit là d'un rythme spatial, d'une danse avec un ordre de poses mais sans cadence imposée. Il s'agit néanmoins toujours de fondre à l'intérieur d'un mouvement à structure continue, un certain nombre d'instants et de poses privilégiés.

\section{Conclusion}

53 Dès lors, si le jeu n'est pas réductible au procès du travail, c'est précisément parce qu'il en constitue une représentation. Il en va de la nature digitale des images vidéoludiques et du caractère autotélique et fictionnel des jeux vidéo, de tendre vers la mise en scène de la réduction d'une quantité d'information. Que ce soit dans une narration linéaire ou dans un monde dit ouvert, le moins que l'on puisse offrir à un joueur, ce sont des objectifs et des moyens pour les accomplir. Même lorsque l'accent est mis sur l'exploration libre, la volonté de faire une expérience kinésique plus contemplative que laborieuse, comme notamment chez Jenova Chen avec Flower, l'agir se fait toujours image d'action, la production, performance réglée dans le but de produire les images.

Plus encore le digital consiste à la lettre en une réduction, au sens où ce n'est pas l'ensemble de la main qui est en jeu, mais bien le doigt. Plus qu'action réelle, le geste digital se fait ainsi représentation de toute action possible par la pression rythmique de boutons. Le joueur est la machine chargée de couper le flux que le programme produit, pour lui donner du sens, ou, plus simplement encore, y remettre de l'ordre. Mais en ce 
que le bruit n'est pas véritable puisque le désordre s'accompagne toujours des moyens et techniques capables de le résoudre (l'interface), le joueur ne coupe pas le flux selon son bon plaisir, il fait fonctionner la machine à images. Son influence directe discontinue (ciseaux) doit s'accorder avec les instants ou les points privilégiés (pointillés) du flux apparemment continu d'images reçues. Ainsi, l'exploration de tout espace vidéoludique, même sans enjeux bien déterminés, consiste déjà en un déploiement d'images qui forment bien plus qu'un espace, un territoire, c'est-à-dire quelque chose à territorialiser, à rythmer. Le joueur inexpérimenté s'y perd et s'y cogne, celui accompli déroule le plus harmonieusement possible les images virtuellement présentes. Au-delà de denrées digitales, ou de bons points, c'est le jeu que le joueur doit s'approprier; ce dernier travaille à produire l'illusion d'un agir efficace pour faire territoire avec les images.

\section{BIBLIOGRAPHIE}

CAILLOIS R. (1967 [1958]), Les jeux et les hommes : le masque et le vertige, Paris, Gallimard.

DELEUZE G. et GUATTARI F. (1972), L'Anti-CEdipe, Paris, Les Éditions de Minuit.

DELEUZE G. (1983), Cinéma I. L’image-Mouvement, Paris, Les Éditions de Minuit.

HEGEL G. W. F. (1998 [1835]), Esthétique tome I, Paris, Flammarion.

KÜCKLICH J. (2005) « Precarious playbour: Modders and the digital games industry », The Fibreculture Journal, $\mathrm{n}^{\circ} 5$

MANOVICH L. (2006 [2001]), Le langage des nouveaux médias, Paris, Les presses du réel

MARX K. (1872), Le Capital, Paris, Maurice Lachâtre

SERRES M. (1980), Le parasite, Paris, Grasset

SMITH A. (1999 [1776]), Recherches sur la nature et les causes de la richesse des nations, [Paris, GF-Flammarion.

ZEIGARNIK B. (1927). « Das Behalten erledigter und unerledigter Handlungen ». Psychologische Forschung, 9, 1-85.

\section{NOTES}

1. Le critère de notre corpus est donc entièrement réglé sur la capacité des jeux vidéo à questionner notre hypothèse : à savoir le travail en tant que composante essentielle de l'image vidéoludique. Nous ne nous limitons pas en ce sens à un genre ou un type de jeu, ni à un mode de production. Nous excluons seulement, toujours dans le but de rester au plus près de notre hypothèse, toute composante agonale (non pas le multijoueur, mais le joueur contre joueur) qui risquerait de rajouter beaucoup de confusion. 
2. Soit A (tas de bois) et A' (table), nous appelons «travail» l'ensemble des opérations qui concourent à la transformation de $\mathrm{A}$ en $\mathrm{A}^{\prime}$. La séquence ordonnée est l'ensemble articulé des tâches $\left(\mathrm{A} \rightarrow \mathrm{A}\right.$ " $\left.\rightarrow \mathrm{A}^{\prime \prime} . . . \rightarrow \mathrm{A}^{\prime}\right)$ qui permet cette réalisation.

3. The $48 \mathrm{~h}$ Global Game Jam, 2009.

4. Nous ne voulons pas dire par là que ce soit toujours aussi facile, mais que c'est possible, comme l'attestent nombre de free-to-play disponibles pour téléphones ou tablettes.

5. http://edmundmcmillen.blogspot.fr/2009/02/avgm-retrospective.html, consulté le 15 novembre 2018.

6. On pourrait nous objecter que la motivation de ces joueurs acharnés n'est pas si aisément caractérisable en tant que compulsion. En effet, c'est peut-être une volonté de comprendre le jeu, un certain humour, etc. qui les déterminent à agir. Mais peu importe les diverses causes que nulle analyse ne saurait réduire, il n'en reste pas moins que c'est parce que le jeu promet quelque chose de plus, un progrès, que le joueur clique.

7. to loot : piller, prendre un butin. Vocable courant pour désigner le fait de trouver un objet dont les règles d'apparition sont aléatoires (on trouve telle hache magique une fois sur trente, sur tel dragon par exemple) et de se l'approprier.

8. Une chaîne du réseau social Twitch, TwitchPlayPokemon, permettait en 2013 de jouer collectivement au jeu de rôle de Nintendo Pokémon par l'intermédiaire d'un script sur le navigateur, tout se passant comme si quelques 800 personnes appuyaient simultanément sur des touches différentes de la Game Boy, jour et nuit. Cela a demandé plusieurs mois et quelques ajustements pour passer quelques endroits difficiles, mais le jeu a bien été fini de cette manière.

9. http://mmorpg-astuce.over-blog.com/article-se-faire-entre-20-et-40m-de-kamas-parsemaine-en-etant-mono-compte-120569035.html, consulté le 15 novembre 2018.

10. Les mouvements des sprites non-joueur peuvent être en réalité fonction de variables fort diverses, charge au joueur de comprendre comment les pièges fonctionnent.

\section{RÉSUMÉS}

Les jeux vidéo, en ce qu'ils prescrivent des opérations à accomplir dans un certain ordre pour atteindre quelque produit, ont de grandes affinités avec le schéma de production du travail. Le joueur y trouve toutes les émotions liées à l'exécution d'une tâche, la frustration de l'échec et la gratification du succès, à grand renfort de sons et d'images. Le plaisir lié à l'accumulation de richesses digitales fournit une image rassurante de la progression et stimule le système de récompense/renforcement. Au-delà des jeux qui exploitent ce trait de manière abusive, et où l'essentiel consiste à cliquer pour recevoir des récompenses, nous verrons que la production désirante est une composante essentielle du jeu vidéo. L'objet du désir, ce vers quoi tend la production, étant lui-même une image, une illusion, la production du joueur n'est pas de l'ordre d'un objet ou d'une œuvre, mais d'une intensité, d'une quantité intensive. Au travers des images, le joueur éprouve, la représentation d'un devenir, d'un passage. Bien plus qu'il exécute une suite d'actions, il perçoit une suite d'actions dont il est responsable, éprouve une intensité qu'il altère lui-même.

Video games, as they prescribe operations to be performed in a certain order to achieve some product, have great affinities with work. The player finds all the emotions related to the execution of a task, frustration of failure and gratification of success, punctuated with great 
reinforcement of sounds and images. The pleasure of accumulating digital rewards provides a reassuring image of progression and stimulates the reward / reinforcement system. Beyond the games that exploit this game design trick in an abusive way and where the essential thing is to click to receive rewards, we will see that the desiring production is an essential component of video game. The object of desire, that towards which production tends, being itself an image, an illusion, the production of the player is an intensive quantity. Through the images, the player experiences, the representation of a becoming, of a passage. Much more than he executes a series of actions, he perceives a series of actions for which he is responsible, experiencing an intensity that he himself distorts.

INDEX

Keywords : work, aesthetics, philosophy, game, video game

Mots-clés : travail, esthétique, philosophie, jeu, jeu video

\section{AUTEUR}

\section{STELLO BONHOMME}

Université de Nice Sophia-Antipolis 